\title{
Estrategias y competencias profesionales de estudiantes de Grado en Bellas Artes para mejorar la visibilidad de sus proyectos finales en plataformas online y redes sociales \\ Strategies and Professional Competences of Fine Arts Degree Students: Improving the Visibility of the Students' Final Projects on Online Platforms and Social Networks
}

\author{
José Gómez-Isla, Gema López Pérez, Julio Pérez Cornejo, Juan Sebastián González Rodríguez, Carmen González \\ García, Cristina Parellada-Bezares and Felicidad García-Sánchez \\ pepeisla@usal.es, cmngonzalez@usal.es, glopez@usal.es, juliopc@usal.es, Juansebastian@usal.es, cristinaparellada@usal.es, \\ felicidadgsanchez@usal.es
}

Departamento de Historia del Arte - Bellas Artes

Facultad de Bellas Artes. Universidad de Salamanca

Salamanca, España

\begin{abstract}
Resumen- El presente trabajo presenta los procesos, metodología y resultados de un proyecto de innovación docente realizado en la Facultad de Bellas Artes de la Universidad de Salamanca con el propósito de dar visibilidad profesional en plataformas online a los proyectos finales de los estudiantes generados tanto en su Trabajo Fin de Grado como en asignaturas de proyectos de último curso de Grado. Las redes sociales (Instagram, Facebook) posibilitan que el trabajo artístico tenga mayor capacidad de penetración que nunca para llegar a los sectores estratégicos y profesionales adecuados. Es pues una oportunidad para dar a conocer las propuestas de nuestros estudiantes en tanto que jóvenes artistas en formación a punto de salir al mercado laboral. Se ha realizado una labor de aprendizaje cooperativo entre alumnos y profesores, con jornadas de formación para conseguir una mayor eficacia comunicativa, intercambiando ejemplos significativos de trabajos y perfiles artísticos diseñados específicamente para redes sociales. También se ha intentado que la Facultad no sea solo un centro educativo al uso, sino un altavoz visual que se haga eco del trabajo de los estudiantes a través de una galería de proyectos destacados en su página web y su perfil institucional de Facebook e Instagram.
\end{abstract}

Palabras clave: Creación artística, visibilización online, aprendizaje cooperativo, profesionalización creativa.

Abstract- The present paper presents the processes, methodology and results of a teaching innovation project carried out in the Faculty of Fine Arts of the Universidad de Salamanca. The aim of this project is to improve professional visibility on online platforms to the Final Projects of our art students in various subjects of their final year projects. Social networks (like Instagram, Facebook) have made it possible for artistic work to have more penetration capacity than ever before in order to reach the right strategic and professional sectors and make students' proposals known as young artists in training. These students need these visibility tools to present themselves in the labour market. There has been a work of cooperative teaching-learning between students and teachers, with training interactive sessions to achieve greater communication effectiveness, and exchanging significant examples of work and artistic profiles designed specifically for social networks. Similarly, it has been intended that the Faculty was not only an educational centre, but a visual speaker that echoes the work of students through a gallery of featured projects on its website and its institutional profile of Facebook and Instagram.

Keywords: Artistic creation, online visibility, cooperative teaching \& learning, creative professionalization

\section{INTRODUCCIÓN}

Las redes sociales y el networking se han convertido en piezas clave de comunicación no sólo para el tiempo de ocio de las jóvenes generaciones, sino sobre todo para su emprendimiento y su inserción en el mundo laboral (Collado Durán, 2019).

Lo que comenzó siendo un medio de entretenimiento social en pleno desarrollo de la llamada Sociedad Red (Castells, 2003), se ha convertido en pocos años en herramienta casi imprescindible para que personas con intereses comunes puedan conectarse fácilmente entre sí a nivel profesional. Como consecuencia de una cultura cada vez más globalizada, resulta particularmente difícil discriminar y seleccionar la información útil para nuestros objetivos. Pero lo que resulta aún más complicado es conseguir cierta visibilidad en estas redes sobre lo que uno hace como prosumidor -es decir, no sólo como consumidor de información, sino también como productor de contenidos audiovisuales- (Toffler, 1979).

El trabajo creativo de nuestros estudiantes (artistas en formación) pasa durante el Grado en Bellas Artes por fases de gran intensidad debido a los requerimientos académicos del actual plan de estudios. La realización de los Trabajos de Fin de Grado en Bellas Artes supone un momento decisivo de su desarrollo creativo. Con este trabajo culminan una fase de su formación universitaria y, al mismo tiempo, supone un punto de 
inflexión hacia una nueva etapa de madurez y consolidación de lo aprendido en entornos profesionales donde tendrán que tomar sus propias decisiones.

Para afianzar el carácter profesionalizante de este Trabajo Fin de Grado (a partir de ahora TFG), durante el curso 20182019 un grupo de profesores y personal investigador en formación de la Facultad de Bellas Artes de la Universidad de Salamanca (a partir de ahora USAL) hemos desarrollado un proyecto de innovación docente incluyendo para ello el concurso de estas redes sociales. El objetivo principal consiste en promocionar los trabajos creativos de nuestros estudiantes más allá de las paredes de la Facultad y que constituyan así un primer paso para la adquisición de competencias profesionales. Para ello se ha fomentado el uso de herramientas que les permitan dar visibilidad a sus proyectos artísticos de TFG y a su competencia profesional, fundamentalmente a través de las plataformas online y las redes sociales.

La presente comunicación da cuenta de los resultados obtenidos con este proyecto. Para ello hemos analizado algunos ejemplos significativos de diferentes formas de elaboración de perfiles artísticos profesionales en redes sociales, sobre todo, Facebook e lnstagram, por ser para el estudiante de Bellas Artes una de las mejores vías para dotar de visibilidad y difusión a su trabajo creativo. El análisis realizado ha ofrecido también una comparativa entre herramientas de presentación visual de dichos perfiles profesionales y artísticos y los proyectos en el marco de las Bellas Artes (Costa, 2015).

\section{CONTEXTO}

La asignatura que comprende el "Trabajo Fin de Grado" en el actual plan de Estudios de Grado en Bellas Artes de la USAL tiene una carga muy importante (de 18 créditos ECTS), y es requisito imprescindible para cualquier estudiante que supere el resto de asignaturas del plan de estudios de cuatro cursos que conforma el Grado en Bellas Artes. Por tanto, el seguimiento, acompañamiento y orientación de dichos estudiantes durante el desarrollo y evolución de estos trabajos por parte de los profesores, en tanto que tutores responsables de dichos TFG, supone una parte irrenunciable de nuestro mandato docente. De igual forma, las asignaturas obligatorias de cuarto curso de Grado en Bellas Artes, "Idea, Concepto y Proceso en la Creación Artística III", "Metodología del Proyecto II" y "Lenguajes y Técnicas en la Producción Artística III", además de las asignaturas optativas de Diseño Gráfico, también están directamente relacionadas con la producción de proyectos artísticos y metodologías creativas de carácter profesional.

El proyecto de innovación docente del que nace esta comunicación no pretendía que los trabajos de $4^{\circ}$ curso se convirtiesen en proyectos finales al uso, circunscritos únicamente a la tutela y el desarrollo exclusivo dentro del aula. Nuestro objetivo principal ha perseguido que, al tratarse de proyectos finales dentro de un ciclo de formación universitario en enseñanzas artísticas, tanto el trabajo del TFG como el resto de proyectos de asignaturas de especialización de último curso, constituyan un primer paso para la adquisición de competencias profesionales en el siempre difícil mundo laboral que espera a nuestros estudiantes tras superar su último año académico.

Por todo ello, se ha procurado que el estudiante adquiera una metodología de trabajo adecuada, no sólo para el desarrollo y producción de sus proyectos finales, sino que dicho desarrollo le sirva también como plataforma promocional para dar visibilidad mediante plataformas online y redes sociales al grado de maestría y competencia profesional alcanzados.

Los objetivos generales que ha perseguido este proyecto de innovación docente han sido los siguientes:

1. Adquisición de una competencia comunicativa y visual adecuadas a través de plataformas online para dar visibilidad al propio trabajo de creación del estudiante en su proyecto final del estudiante a lo largo de su último año de grado en Bellas Artes.

2. Aprendizaje (mediante ejemplos significativos) de las diferentes formas de elaboración de un perfil profesional en las redes sociales, sobre todo Facebook e Instagram, por ser actualmente para el estudiante de Bellas Artes la mejor forma para difundir y posicionar su trabajo de creación.

3. Adquisición de habilidades por parte del estudiante para la elaboración de un porfolio o book profesional dinámico, coherente y ordenado relacionado con su Trabajo Fin de Grado y sus proyectos finales, y que le sea útil de cara a su futuro laboral tras finalizar sus estudios de grado.

4. Analizar y comparar distintas estrategias de presentación visual de perfiles profesionales y artísticos y de proyectos en el marco de las Bellas Artes.

5. Que el estudiante sepa identificar la metodología de trabajo más adecuada para difundir su TFG y el resto de sus proyectos artísticos y conseguir así una mejor penetración en el mercado laboral tras finalizar sus estudios de grado.

6. Elaboración de un plan de trabajo estratégicamente diseñado para conseguir presentaciones innovadoras, coherentes y eficaces en redes y plataformas online tanto del perfil profesional del alumno/a como de su producción creativa reciente.

7. Generar sinergias adecuadas con instituciones y empresas culturales para que los TFG y los proyectos finales de los estudiantes sean presentados en sociedad, tanto en Centros de Arte y ámbitos profesionales de creación e investigación artística, como en entornos virtuales donde dichas instituciones estén directamente implicadas.

Además de los objetivos generales relacionados con la adquisición de competencias relativas al desarrollo profesional del estudiante, hemos identificado otros objetivos transversales que redundan en un mejor funcionamiento del trabajo coordinado entre los profesores implicados en el proyecto:

a) Creación de sinergias e intercambio de experiencias entre los profesores pertenecientes a distintas áreas de conocimiento para enriquecer el trabajo coordinado en la tutela y seguimiento de los TFG y los proyectos artísticos de los estudiantes, aunque la tutela de cada estudiante se ejerza de manera individualizada.

b) Consolidar y mejorar el trabajo de coordinación entre tutores de TFG y proyectos finales en asignaturas de $4^{\circ}$ curso para que los objetivos sean transversales y no se reduzcan a áreas de conocimiento acotadas dentro del grado en Bellas Artes, como si de compartimentos estancos se tratase.

c) Compartir metodologías de desarrollo y visualización a través de las redes sociales desde las distintas perspectivas de especialización a la que pertenezca cada docente. De 
esta manera, el proceso de tutela del TFG puede entenderse como una propuesta colaborativa en forma de vasos comunicantes entre disciplinas, ámbitos de trabajo y perfiles profesionales diversos.

d) Generar una guía de recursos para compartirla con otros profesores cuya docencia se adscriba al Grado en Bellas Artes para que puedan aplicarla o personalizarla en el futuro en su tarea de tutela y seguimiento de los TFG y los proyectos finales de nuestros estudiantes.

\section{DESCRIPCIÓN}

La labor de coordinación del proyecto entre los docentes implicados se acometió durante el pasado curso académico 2018-19 en forma de reuniones periódicas quincenales, especialmente en su fase de planificación (enero de 2019), para diseñar las estrategias metodológicas en el aprendizaje y emprendimiento del estudiante que hemos intentado promover.

La fase operativa se ha prolongado durante el periodo de ejecución y seguimiento del TFG y los proyectos finales de los estudiantes para coordinar a los docentes implicados en este proyecto de innovación. El proyecto se ha coordinado de forma sincrónica con los tiempos y fases del proceso de maduración y ejecución del trabajo del estudiante, así como sus estrategias comunicativas en redes. De este modo, el estudiante procedía adecuadamente a la presentación digital, difusión online y promoción en redes de los resultados finales durante el periodo de tutela y elaboración del TFG y el resto de proyectos artísticos de último curso de Grado.

Las fases sucesivas de este proyecto de innovación docente han sido las siguientes:

- Primera fase. Recopilación colectiva de la literatura científica y de las experiencias precedentes en planes de trabajo con características similares (siempre en el ámbito de la creación artística) que han inspirado y mejorado las propuestas que cada miembro del equipo docente ha podido aportar en una segunda fase.

- Segunda fase. Reuniones del equipo de innovación docente para poner en común las distintas estrategias de promoción y difusión del trabajo de los estudiantes a través de plataformas online y redes sociales (Instagram y Facebook fundamentalmente).

- Tercera fase. Periodo de entrenamiento a través de seminarios prácticos de especialización y visibilización, en forma de jornadas y charlas formativas de expertos en materia de comunicación digital. De esta forma, el equipo docente y los estudiantes implicados fueron capaces de conocer y manejar de primera mano las distintas metodologías de trabajo en plataformas digitales y redes sociales para presentar los resultados de los TFG o de los proyectos finales tutelados.

- Cuarta fase. Presentación colectiva a los estudiantes de $4^{\circ}$ curso de la planificación y las estrategias metodológicas que se querían implementar para maximizar la difusión y promoción de sus proyectos artísticos en redes sociales y plataformas online, acordes a los objetivos ya mencionados de cara a una mejor inserción en el mercado laboral.

- Quinta fase. Fase operativa $\left(2^{\circ}\right.$ cuatrimestre del curso 2018-19) donde los estudiantes comenzaron a subir a plataformas y redes sociales los resultados y contenidos elaborados durante el periodo de producción de su TFG y otros proyectos finales.

- Sexta fase. Análisis de los resultados obtenidos y discusión colectiva de los mismos con el equipo docente para detectar los problemas y acometer las posibles soluciones antes del final de curso y de la presentación del propio TFG y de los proyectos finales de asignaturas de $4^{\circ}$ curso.

- Séptima fase. Evaluación del proyecto haciéndolo coincidir con el periodo de entrega y evaluación de los propios TFG y del resto de proyectos artísticos en asignaturas de $4^{\circ}$ curso.

- Octava fase. Elaboración de la memoria final con los resultados del proyecto ejecutado.

La primera y segunda fase fueron casi consecutivas y se resolvieron en las tres primeras semanas de inicio del segundo cuatrimestre, antes de la asignación de los tutores a los TFG correspondientes de los estudiantes. Dichas fases tuvieron lugar entre mediados de enero y principios de febrero de 2019.

La tercera y cuarta fase se acometieron durante los meses de febrero y marzo de 2019.

La quinta fase se acometió entre abril y mayo de 2019.

La sexta fase, casi consecutiva respecto a la quinta, permitió detectar en tiempo real los problemas surgidos con la implementación del proyecto, abordando así las posibles soluciones y correcciones (abril-mayo de 2019).

La séptima fase de evaluación, discusión de resultados y conclusiones del proyecto se acometió a lo largo del mes de junio de 2019.

La octava y última fase, consistente en la elaboración de la memoria final con los resultados obtenidos sobre la experiencia del proyecto, se desarrolló durante la segunda quincena de junio de 2019.

\section{Resultados}

Tal y como contemplaba el plan de trabajo del proyecto, se llevaron a cabo una serie de acciones durante el transcurso del mismo que permitieron evolucionar a nuestros alumnos en el desarrollo de sus perfiles profesionales en redes y acometer un plan estratégico para dar visibilidad a sus proyectos artísticos de TFG y proyectos finales de otras asignaturas de $4^{\circ}$ curso, siempre con vocación profesional.

Una de las primeras acciones consistió en una búsqueda de expertos en comunicación online y directivos de agencias de comunicación y marketing. Se pretendía así conocer de primera mano las estrategias más adecuadas para diseñar perfiles profesionales, construir una "marca personal" y dar la mejor visibilidad a los proyectos artísticos en redes sociales (esencialmente Facebook e Instagram).

Tras esa búsqueda y su idoneidad en materia de producción artística, se invitó a dos de estos profesionales a participar en unas jornadas de formación e intercambio de experiencias destinadas tanto a estudiantes como a profesores para poder diseñar y desarrollar después, en una segunda fase, lo que se conoce como "marca personal" (Pérez Ortega, 2014), es decir, la construcción de perfiles creativos profesionales y la producción de proyectos destinados a generar la mayor 
visibilidad posible en estas redes sociales. En concreto, los dos profesionales invitados fueron Jesús Herrero (director de Take Away Agency) y Álex Sánchez (Creador y blogger de "Tiempo de Publicidad" y Head of Social Media y Digital Copywriter en la agencia $M c C A N N$ ).

El encuentro con estos expertos se denominó "Jornadas de visibilización profesional de proyectos artísticos en redes sociales y plataformas online". Se celebró en dos sesiones el 18 de marzo de 2019 en el aula B.2. de Facultad de Bellas Artes de la Universidad de Salamanca. (Ver figura 1)

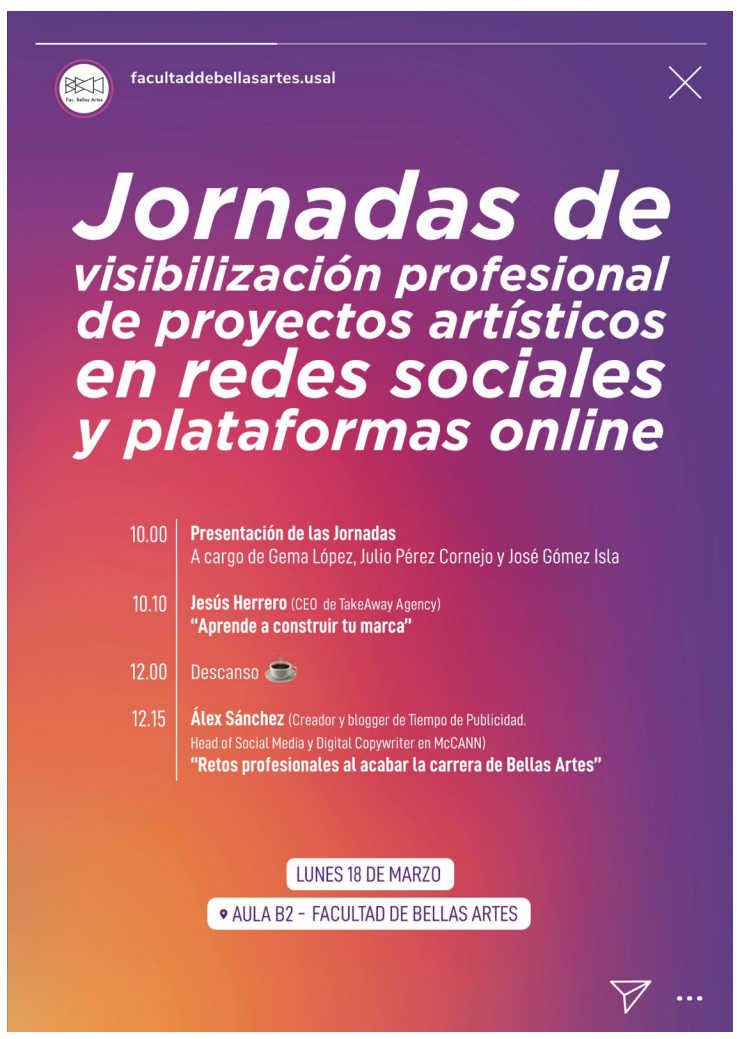

Figura 1. Cartel anunciador de las jornadas de visibilización

En su intervención, Jesús Herrero abordó las vicisitudes del aprendizaje a la hora de construir una "marca personal" eficaz, diseñando el perfil más adecuado, conociendo al público objetivo a quien van dirigidos los proyectos artísticos de los estudiantes, y analizando dónde, cómo y con quién conectar. El objeto de análisis se trató mediante ejemplos prácticos de autores consolidados que han triunfado al dar visibilidad y prestigio a su trabajo creativo (Deckers and Lacy, 2013).

Por su parte, Álex Sánchez abordó los retos profesionales con los que se enfrenta el estudiante al acabar su carrera de Bellas Artes, en concreto, lo que la comunidad profesional debería saber del estudiante, lo que el propio estudiante debería saber del sector profesional en el que se enmarca su trabajo, así como las estrategias más adecuadas para presentar y difundir adecuadamente en dicho sector las obras que el estudiante genera en su TFG y sus proyectos finales.

Tras la celebración de las jornadas, se administró una encuesta suficientemente representativa entre los estudiantes asistentes. Esta encuesta fue diseñada con la herramienta digital Mentimeter (una aplicación ya testada y utilizada con buenos resultados en otro proyecto anterior). El objetivo de la encuesta consistía en conocer el grado de satisfacción de los estudiantes durante las jornadas y el grado de implementación de las recomendaciones de los profesionales invitados a dichas jornadas a la hora de diseñar su perfil profesional y dar visibilidad a sus propios proyectos. El resultado de las encuestas, respondidas mediante una app sencilla (www.menti.com), a la que los estudiantes podían acceder a través de sus propios teléfonos móviles, resultó satisfactorio y muy orientativo. Entre las preguntas formuladas, se les planteó si tenían ya diseñado un perfil "profesional" para visibilizar sus proyectos artísticos en redes y online, a lo que la práctica totalidad de los estudiantes contestó que sí (ver tabla 1).

Tabla 1. Encuesta realizada al alumnado sobre las jornadas

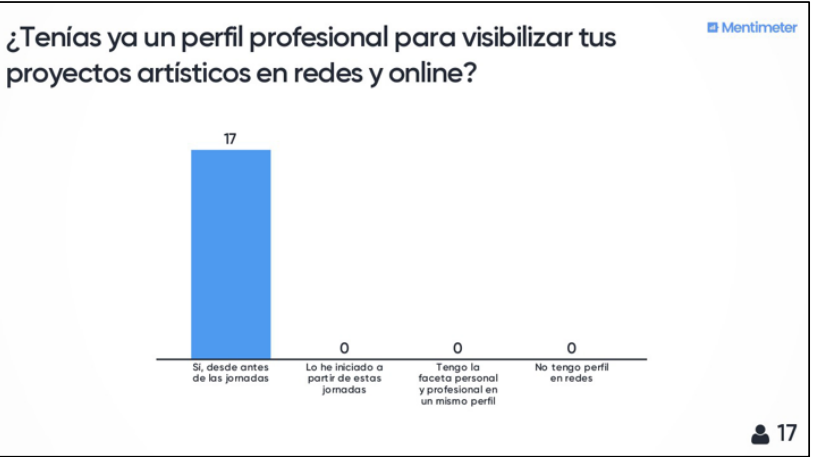

Otra pregunta intentaba averiguar el grado de utilidad de esas jornadas de formación e intercambio y si les habían servido a los estudiantes para replantearse las estrategias de visibilización de sus proyectos artísticos en redes, a lo que más de la mitad de estudiantes respondió que "bastante" (4) en una escala de Likert entre 1 y 5 (ver tabla 2).

Tabla 2. Encuesta realizada al alumnado sobre las jornadas

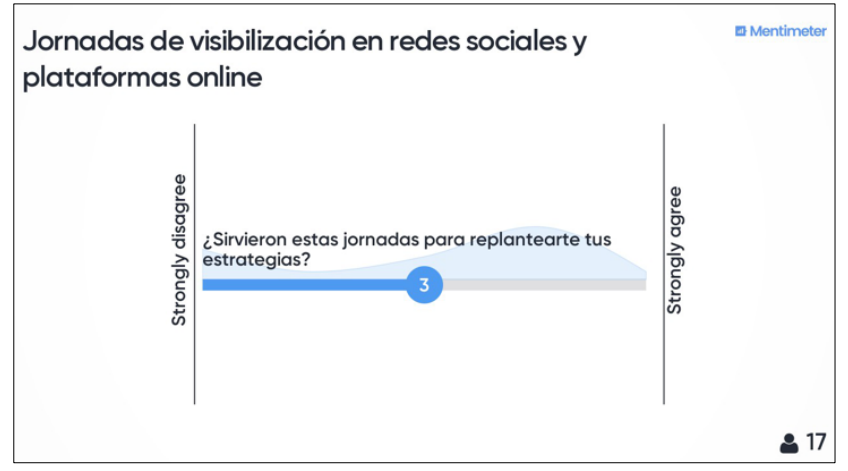

Otra pregunta indagaba en el grado de satisfacción de actividades como esta, realizadas en la Facultad en los últimos años. Mayoritariamente los estudiantes contestaron que sí les resultaron satisfactorias para sus propios intereses (ver tabla 3).

Tabla 3. Encuesta realizada al alumnado sobre las jornadas

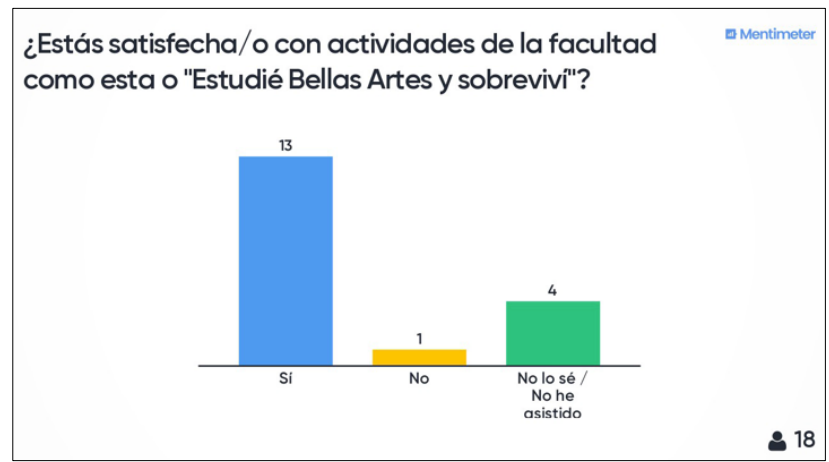


Por último, se formuló una pregunta abierta proponiendo que realizasen sugerencias de mejora para esta y otras actividades de formación similares. Propusieron actividades enfocadas mayoritariamente a las salidas profesionales de la carrera, sobre todo aquellas vinculadas con disciplinas concretas (dibujo, pintura, diseño, ilustración, animación). En algún caso se propuso una mejor orientación sobre másteres especializados, tras la finalización de sus estudios de Grado (ver tabla 4).

Tabla 4. Encuesta realizada al alumnado sobre las jornadas

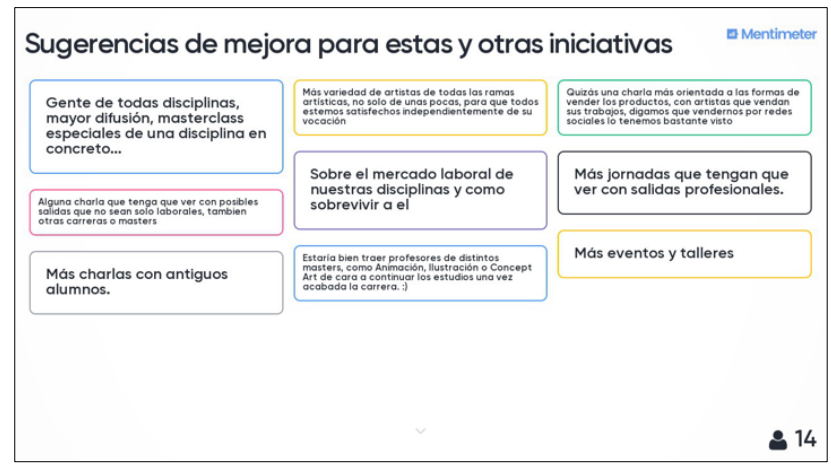

En una tercera fase, cuando los TFG estaban suficientemente avanzados, se buscó la implicación de los estudiantes para que subiesen imágenes de sus proyectos a las redes sociales. Se pretendía así que hallasen los modos más eficaces para subir contenidos en función del proyecto artístico específico, su perfil profesional, su marca personal, así como la incorporación de hashtags, como "\#TFGBBAAUSAL19" que aludiesen no sólo a su trabajo personal, sino también a la institución donde esos trabajos habían sido realizados y tutelados (figuras 2, 3 y 4).
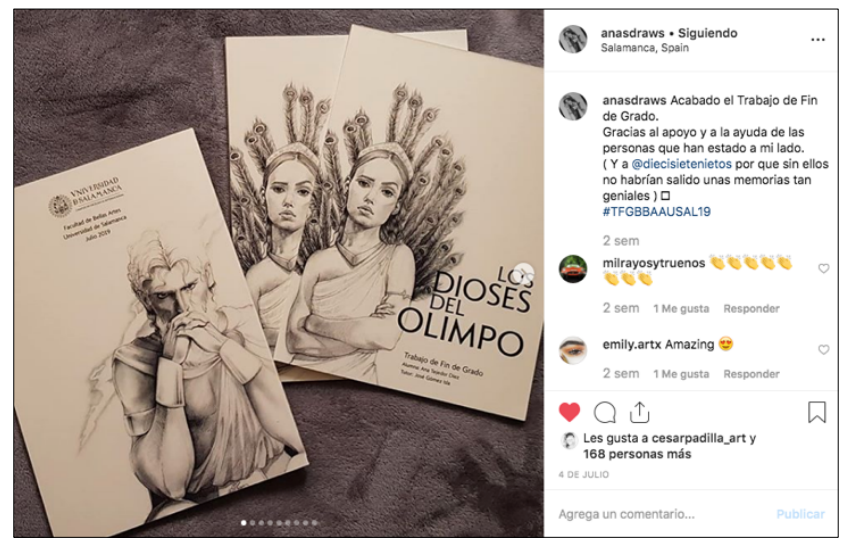

Figura 2. Imagen de Instagram de la alumna Ana Tejedor

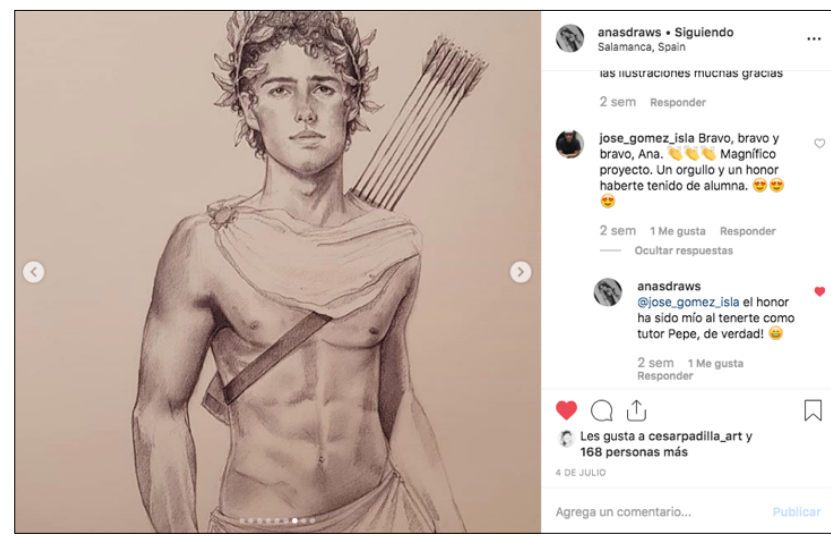

Figura 3. Imagen de Instagram de la alumna Ana Tejedor
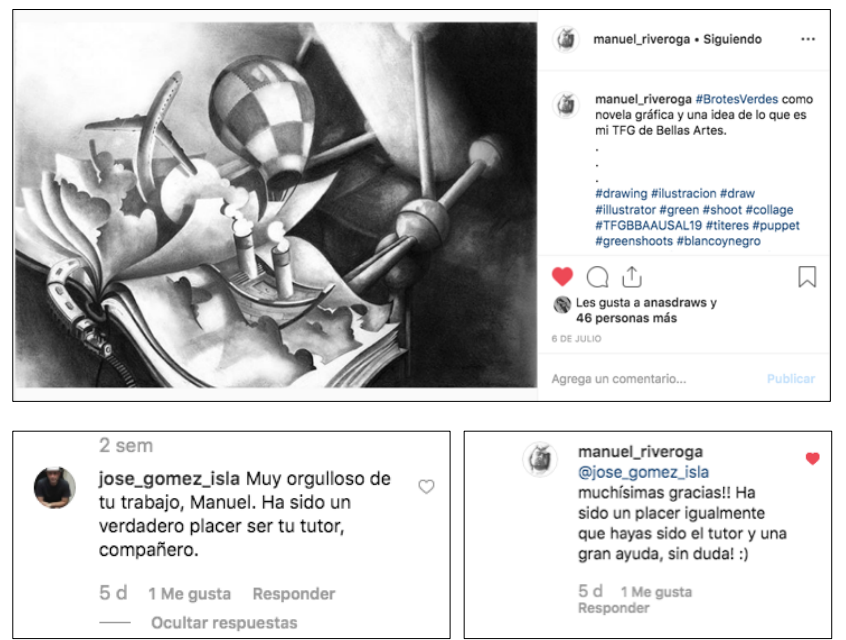

Figura 4. Imagen de Instagram del alumno Manuel Rivero

En una última fase se abordó el rediseño de la web de la Facultad a cargo de profesores de las asignaturas de diseño (con la colaboración de estudiantes matriculados en estas asignaturas). En dicha web se incorporó una "galería de arte" para dar a conocer una selección de proyectos de alumnos de último curso (de TFG y trabajos finales de asignaturas de $4^{\circ}$ ). Se buscaron sinergias entre lo publicado en los perfiles profesionales de los estudiantes en Facebook e Instagram y lo publicado en la página web de la Facultad y otros canales institucionales, como el muro de Facebook o el perfil de Instagram de la propia Facultad de Bellas Artes.

Tras finalizar esa última fase, se realizó una reunión final donde se dieron cuenta de los resultados obtenidos por los estudiantes como la experiencia acumulada entre los profesores tutores. Se elaboraron unas conclusiones finales del proyecto y también algunas sugerencias de mejora para el futuro.

\section{CONCLUSIONES}

Para el estudiante de grado de la Facultad de Bellas Artes ha sido determinante no sólo el aprendizaje de las prácticas artísticas (a lo largo de sus cuatro años de grado), sino también el conocimiento y el uso eficaz de herramientas web, redes y plataformas digitales existentes en los actuales contextos comunicativos para promocionarse profesionalmente y dar visibilidad a sus propios proyectos de creación artística.

De cara a la búsqueda de sus salidas profesionales, ha sido también crucial el aprovechamiento inteligente de las ventajas que ofrecen las plataformas digitales y redes sociales más utilizadas. Los contextos virtuales de la llamada Web 2.0 generan oportunidades inmejorables para difundir sus propuestas artísticas; sirven como carta de presentación para que el estudiante recién salido de la Facultad se dé a conocer en el entorno profesional de la creación contemporánea, lo que le proporciona un acercamiento directo y privilegiado a empresas e instituciones culturales donde promocionar su trabajo.

Las herramientas utilizadas durante esta experiencia de innovación están directamente relacionadas no sólo con la elaboración de proyectos profesionales sólidos en los distintos ámbitos de especialización artística, sino también con la forma de difundir profesionalmente dichos proyectos y darles una visibilidad adecuada en las plataformas online que los softwares $\mathrm{y}$ los dispositivos actuales nos ofrecen. 
Las mejoras detectadas en el aprendizaje de los estudiantes se concretan en los siguientes puntos:

a) Mejora del conocimiento y acceso al mundo profesional y al mercado laboral dentro de su ámbito de especialización.

b) Mejora de las estrategias comunicativas del estudiante para identificar y presentar de forma eficiente y profesional sus propios proyectos artísticos.

c) Mejora en las habilidades de manejo como productores de contenidos audiovisuales a través de las TIC.

d) Mejora en el conocimiento del ámbito profesional que generan las plataformas online y redes sociales.

e) Mejora de los formatos de presentación de sus proyectos artísticos para hacerlos más atractivos y dinámicos a nivel visual en entornos multimedia.

f) Mejora de las estrategias de networking del estudiante para ponerse en relación con otros profesionales del sector en el ámbito de la creación artística.

De igual modo, se ha procurado que los estudiantes tutelados conozcan las estrategias comunicativas y de producción de contenidos multimedia empleadas por el resto de sus compañeros de Grado a quienes también afectaba este proyecto de innovación para lograr sinergias e intercambio de ideas entre ellos a la hora de presentar profesionalmente su trabajo creativo.

Como conclusión final, durante esta experiencia se produjo una relación de estrecha colaboración y aprendizaje fluido de ida y vuelta entre estudiantes y profesorado (como se aprecia en los comentarios vertidos en Instagram entre estudiantes y profesores). De este modo, las competencias y habilidades de cada colectivo han servido para actualizar conocimientos del otro (y viceversa) mejorando así las estrategias de comunicación online de todos ellos. Esta relación de cooperación también generó un mayor "sentido identitario" de los estudiantes y una mayor implicación con la institución universitaria a la que pertenecen (véanse los post de las figuras 2, 3 y 4). La propia Facultad de Bellas Artes también ha puesto recientemente en valor el trabajo de los estudiantes a través de su página web oficial (recientemente rediseñada) incorporando una selección de proyectos en una galería destacada, y a través de las redes sociales de la propia Facultad (Facebook e
Instagram). Con ello también se ha dado a conocer el trabajo cooperativo de enseñanza-aprendizaje entre profesores y estudiantes dentro de la propia institución. Por último, se han actualizado y perfilado estratégicamente los "hashtag" en dichas redes para aumentar así la visibilidad institucional de los trabajos y proyectos presentados por los estudiantes de Bellas Artes entre la comunidad artística a quien van dirigidos.

\section{AgRADECIMIENTOS}

El equipo de trabajo del presente Proyecto de Innovación Docente quiere agradecer expresamente al Vicerrectorado de Docencia de la Universidad de Salamanca la financiación recibida a través del "Programa de Ayudas a Proyectos de Innovación y Mejora Docente 2018-2019”.

\section{REFERENCIAS}

Castells, M. (1997). La era de la información. Vol. 1. La sociedad red. Madrid, España: Alianza.

Collado Durán, E. (2019). El mundo cambia, ¿y tú? Claves para diseñar tu futuro profesional en plena era digital. Barcelona, España: Planeta.

Costa, N. (2015). Emprender tu marca personal. Barcelona, España: Profit Editorial.

Deckers, E., y Lacy, K. (2013). Branding personal. Cómo usar las redes sociales para promocionarte. Madrid, España: Anaya.

Ferrazzi, K., y Raz, T. (2015). Nunca comas solo. Networking para optimizar tus relaciones personales, Barcelona, España: Profit Editorial.

Pérez Ortega, A. (2014). Marca personal. Cómo convertirse en la opción preferente. Madrid, España: ESIC Editorial.

Peter, T. (2005). 50 Claves para Hacer de Usted una Marca. Cincuenta maneras de transformarse, dejando de ser un "empleado" para convertirse en una marca que comunique a gritos distinción, compromiso y pasión. Barcelona, España: Ediciones Deusto.

Toffler, A. (1980), The Third Wave, New York, Estados Unidos: Bantam Books. 